\title{
Cholecystokinin Measurement
}

National Cancer Institute

\section{Source}

National Cancer Institute. Cholecystokinin Measurement. NCI Thesaurus. Code C74850.

The determination of the amount of cholecystokinin hormone present in a sample. 